\title{
Stenotrophomonas maltophilia bacteremia in children - A 10-year analysis
}

\author{
Ayşe Büyükcam ${ }^{a}$ M.D., Asiye Bıçakcıgil MD, Prof. Dr. Ali B. Cengiza, Prof. Dr. Banu Sancak, \\ Prof. Dr. Mehmet Ceyhan and Prof. Dr. Ateş Kara
}

\begin{abstract}
Stenotrophomonas maltophilia is a multidrug-resistant, Gramnegative, and biofilm-forming pathogen. Information is limited concerning S. maltophilia bacteremia in children. Clinical data and microbiological test results collected in a tertiary children's hospital over a ten-year period were reviewed. Children 0-18 years old who had positive clinical specimen, blood and/or catheter cultures were included. We identified 20 S. maltophilia isolates from 12 pediatric patients with confirmed infections. The median age was 28 months (range: 3.1-187.3). The rate of previous use of antimicrobial therapy was $83 \%$. The median antibioticnumber was 3 (range: $0-7$ ) within 30 days prior to onset of $S$. maltophilia bacteremia. Catheter related infection was the main infectious source $(66.6 \%)$. The mortality rate was $33.3 \%$. The death of two non-survivors was associated with pneumonia. S. maltophilia should be considered a breakthrough agent for bacteremia in children with underlying disease exposed to broad-spectrum antibiotics during long-term hospitalization. Key words: bacteremia, Stenotrophomonas maltophilia, catheter related infection, child, pneumonia.
\end{abstract}

http: / / dx.doi.org/ 10.5546/ aap.2020.eng.e317

To cite: Büyükcam A, Bıçakcıgil A, Cengiza AB, Sancak B, et al. Stenotrophomonas maltophilia bacteremia in children - A 10-year analysis. Arch Argent Pediatr 2020;118(3):e317-e323.

\section{INTRODUCTION}

Stenotrophomonas maltophilia is an aerobic, nonglucose-fermenting, Gram-negative, motile, and biofilm-forming bacterium ${ }^{1}$ that has been isolated from soil, water, plant material, and humans and animals. It has the ability to colonize different sites, like epithelial cells of the respiratory tract

a. Pediatric Infectious Diseases Unit, Department of Pediatrics, Hacettepe University Faculty of Medicine, Ankara, Turkey.

b. Department of Medical Microbiology, Hacettepe University Faculty of Medicine, Ankara, Turkey.

E-mail address:

Ayşe Büyükcam, M.D.: dr.aysebaktir@gmail.com

Funding: None.

Conflict of interest: None.

Received: 6-16-2019

Accepted: 10-17-2019 and surfaces of medical devices. ${ }^{2}$ S.maltophilia was initially thought to be of low level pathogenicity; however, recent studies have identified it as an important opportunistic and nosocomial pathogen that is now observed with increasing frequency. ${ }^{2}$ This is particularly frequent among patients who are immunosuppressed or who have an underlying serious medical condition with high morbidity and mortality.

Nosocomial S.maltophilia infections are common, but community-acquired pathologies have also occasionally been reported. ${ }^{2}$ The most common clinical manifestations of $S$. maltophilia infection are pneumonia and bacteremia, ${ }^{3}$ with endocarditis, or central nervous system, ocular, urinary tract, bone and joint, skin and soft tissue, or gastrointestinal tract infections, occurring less often. It is occasionally associated with septic shock in critically ill and immunosuppressed patients. ${ }^{1}$

The treatment of S. maltophilia infections is a significant management challenge. ${ }^{1,2} S$. maltophilia exhibits intrinsic resistance to many available broad-spectrum antibiotics. ${ }^{4}$ Consequently, an appropriate antimicrobial therapy is often delayed. ${ }^{1}$

A limited number of case-control studies on S. maltophilia bacteremia have been reported and there are only a few reports describing S. maltophilia bacteremia and its features in the pediatric population. ${ }^{5}$ However, pediatric patients with $S$. maltophilia bacteremia have a high mortality rate, ranging from $6 \%$ to $40 \%$, which is similar to the rate reported in adults. ${ }^{6}$

The objective of the present study was to identify the clinical and microbiological characteristics of pediatric patients with S.maltophilia bacteremia over a 10 -year period in our center.

\section{METHODS}

\section{Patients and Methods}

Study design and setting

This single-center, retrospective, observational study was conducted at Hacettepe University İhsan Doğramacı Children Hospital, which is a 
270-bed, tertiary-care children's referral hospital in Ankara, Turkey. This study was approved by the Institutional Review Board of the Hacettepe University Faculty of Medicine.

\section{Data collection and variables}

This 10-year retrospective study examined the pediatric patients ( $0-18$ years old $)$ who were hospitalized from January 2007 to June 2017 and whose blood and/or catheter cultures were positive for S.maltophilia along with clinical symptoms of systemic infection. Microbiological data (isolation sites, susceptibility results, coisolated or persistent organisms, previous isolation of S. maltophilia within 30 days) were confirmed through the microbiological laboratory information system. Clinical data were collected from electronic medical records and patient charts. Exclusion criteria were age $>18$ years and a positive blood culture without adverse clinical signs or symptoms.

\section{Bacterial identification and antimicrobial susceptibility testing}

All blood samples were taken from peripheral veins and/or a central line. Blood cultures were identified using conventional tests and the BBL Crystal E/NF ID system (Becton Dickinson Microbiology Systems, Cockeysville, Maryland, USA) or a matrix-assisted laser desorption ionization-time of flight mass spectrometry system (BioMerieux, France). Antibiotic susceptibilities were tested using the Kirby-Bauer disc diffusion method, according to the Clinical and Laboratory Standards Institute guidelines. ${ }^{7,8}$

\section{Definitions}

S. maltophilia bacteremia was defined as at least one positive blood culture of S. maltophilia with clinical signs of infection. ${ }^{3}$ The Centers for Disease Control and Prevention criteria were used to define central-line-associated bloodstream infection (CLABSI) ${ }^{9}$ (see Annex for definitions).

\section{Statistical analysis}

Data were analyzed using SPSS Statistics 22.0 (SPSS, Chicago, IL, USA). Descriptive statistics were used to summarize the baseline patient characteristics. The continuous variables for patient characteristics were summarized as median and interquartile range (IQR). Frequency distributions and percentages were calculated for categorical variables.

\section{RESULTS}

We identified 48 S. maltophilia isolates. However, 28 isolates were excluded because of contamination or insufficient clinical data. Finally, only 20 isolates from the 12 patients with clinically documented infection were included. No colonization was evident prior to the onset of infection in these 12 patients. The median age was 28 months (range: 3.1 to 187.3 months). The female-to-male ratio was 1:1. All patients had at least one underlying disease, which were primarily immune deficiency and congenital heart disease. Surgery was performed in four patients and prior antimicrobial therapy was noted in ten patients within 30 days prior to the onset of bacteremia.

The median antibiotic number and the rate of use of carbapenems were 3 (range: 0-7) and $66.7 \%(\mathrm{n}=8)$, respectively, within 30 days prior to onset. In total, five patients were considered immunosuppressed. None of our patients were neonates. Six patients were admitted to the intensive care unit (ICU), and four required mechanical ventilation (Table 1). Total parenteral nutrition (TPN) was administered to two patients at 14 days of onset. Ten patients had medical devices (eight central venous catheters [CVCs], three tracheostomy tubes, and one cardiac pacemaker). Ten patients $(83.3 \%)$ had hospitalacquired infections and two had communityacquired infections (patient 7 and patient 10). The central-line-associated bloodstream infection was the main infectious source (in eight patients). Pneumonia was identified in two patients (patient 3 and patient 11) with S. maltophilia bacteremia. S. maltophilia was also isolated from respiratory specimens from these two patients, in one at the onset of bacteremia and in the other 21 days later. Two patients had bacteremia of unknown origin.

The median value for white blood cell counts was $6900 / \mu \mathrm{L}$ (range: $200-20,100 / \mu \mathrm{L}$ ). Two patients were neutropenic $\left(<500\right.$ cells $\left./ \mathrm{mm}^{3}\right)$ and six were thrombocytopenic $(<150,000 /$ $\mu \mathrm{L}$ ). The median albumin level was $3.4 \mathrm{~g} / \mathrm{dL}$ (range:1.3-4.3 g/dL) and three were under $2.5 \mathrm{~g} /$ $\mathrm{dL}$ at the onset of bacteremia.

Polymicrobial bacteremia occurred in four patients. The pathogens found concurrently with S. maltophilia included Enterococcus faecalis, Streptococcus parasanguinis, Staphylococcus epidermidis, and Enterobacter cloacae. The median length of hospitalization was 60.5 days (range: 5-170 days; IQR: 36.5-75.7 days), and the median length of hospitalization after the positive culture 
result was 21.5 days (range: 1-61 days, IQR: 8.748 days).

The duration of bacteremia ranged from 4 to 25 days, with a median of 8 days. Overall, 4 patients received appropriate empiric antimicrobial therapy. The duration between the first positive blood culture and administration of appropriate treatment was $\leq 72$ hours in only five patients. Of all eight CVCs, five were removed during antibiotic treatment for S. maltophilia. Persistent bacteremia occurred in two patients one who started appropriate therapy at 48-72 hours and the other at $>72$ hours. All occurrences of persistent bacteremia had communityacquired S. maltophilia infection, and no prior antimicrobial therapy was noted within 30 days before onset. The patients who developed persistent bacteremia survived. Four patients had also been diagnosed with sepsis, and three of them experienced septic shock.

The median duration of treatment was 15.5 days (range 0-37 days; IQR: 5.5-22.5 days).
The most frequently used antimicrobial agent was ciprofloxacin (six patients) for S. maltophilia infection. Antibiotic susceptibility testing was performed on all isolates (Table 2).

The mortality rate was $33.3 \%(n=4)$. In three of the four fatal cases, S. maltophilia was regarded to have had a direct role in death, and only one of these patients had received appropriate treatment in the first 24 hour after the onset of bacteremia. Additionally, two of them were associated with pneumonia. All three of the non-survivors due to bacteremia had undergone prior treatment with carbapenem 30 days before S. maltophilia infection.

\section{DISCUSSION}

In the current study, all the patients had at least one underlying disease, including immunosuppressive conditions. The previous rate of use of antimicrobial therapy $(83 \%)$ and the median antibiotic number (3) were high within 30 days prior to onset of $S$. maltophilia bacteremia. The most common previously used antibiotic was

TABLE 1. Patient history of antibiotic use and other clinical characteristics

\begin{tabular}{|c|c|c|c|c|}
\hline Patient & 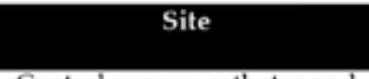 & $\begin{array}{l}\text { Antibiotics within } 30 \\
\text { days prior to onset }\end{array}$ & $\begin{array}{l}\text { Mechanical } \\
\text { ventilation }\end{array}$ & Sepsis/Septic shock \\
\hline 1 & $\begin{array}{l}\text { Central venous catheter and } \\
\text { peripheral blood }\end{array}$ & Ampicillin sulbactam & No & No \\
\hline 2 & Peripheral blood & $\begin{array}{l}\text { Meropenem, } \\
\text { vancomycin, amikacin, } \\
\text { caspofungin }\end{array}$ & Yes & No \\
\hline 3 & Peripheral blood & Meropenem & No & Sepsis \\
\hline 4 & Peripheral blood & $\begin{array}{c}\text { Ceftriaxone, gentamicin, } \\
\text { clindamycin, ornidazole, } \\
\text { fluconazole }\end{array}$ & Yes & Septic shock \\
\hline 5 & $\begin{array}{l}\text { Central venous catheter and } \\
\text { peripheral blood }\end{array}$ & $\begin{array}{c}\text { Meropenem, teicoplanin, } \\
\text { amikacin, } \\
\text { metronidazole, } \\
\text { caspofungin }\end{array}$ & No & Septic shock \\
\hline 6 & $\begin{array}{l}\text { Central venous catheter and } \\
\text { peripheral blood }\end{array}$ & $\begin{array}{l}\text { Meropenem, } \\
\text { vancomycin, } \\
\text { amphotericin B }\end{array}$ & Yes & No \\
\hline 7 & $\begin{array}{c}\text { Central venous catheter and } \\
\text { peripheral blood }\end{array}$ & None & No & No \\
\hline 8 & $\begin{array}{c}\text { Central venous catheter and } \\
\text { peripheral blood }\end{array}$ & $\begin{array}{l}\text { Imipenem, teicoplanin, } \\
\text { amikacin, ciprofloxacin } \\
\text { fluconazole }\end{array}$ & No & No \\
\hline 9 & $\begin{array}{c}\text { Central venous catheter and } \\
\text { peripheral blood }\end{array}$ & $\begin{array}{c}\text { Meropenem, fluconazole, } \\
\text { amphotericin B }\end{array}$ & No & No \\
\hline 10 & $\begin{array}{c}\text { Central venous catheter and } \\
\text { peripheral blood }\end{array}$ & None & No & No \\
\hline 11 & Peripheral blood & $\begin{array}{l}\text { Meropenem, imipenem, } \\
\text { vancomycin, amikacin, } \\
\text { ciprofloxacin, } \\
\text { trimethoprim- } \\
\text { sulfamethoxazole, } \\
\text { fluconazole } \\
\end{array}$ & Yes & Septic shock \\
\hline 12 & $\begin{array}{c}\text { Central venous catheter and } \\
\text { peripheral blood }\end{array}$ & Meropenem, amikacin & No & No \\
\hline
\end{tabular}


carbapenem, followed by aminoglycosides. The occurrences of prolonged hospital stay and ICU stay, the presence of indwelling devices, including CVCs, and the use of immunosuppressive therapy were similar to those described in the literature. ${ }^{1,2}$

S. maltophilia exhibits intrinsic resistance to many available broad-spectrum antibiotics, including carbapenems and aminoglycosides. ${ }^{4}$ The use of broad-spectrum antibiotics, and especially carbapenems, can suppress the overgrowth of many pathogens, and this selection pressure can facilitate overgrowth of S. maltophilia..$^{4}$ In addition, S. maltophilia is an environmentally opportunistic pathogen, and this situation may lead to colonization by this organism. Over the past decades, S. maltophilia infections have become important nosocomial infections, especially those associated with bacteremia. ${ }^{1,2}$

Another risk factor for S. maltophilia infection is long-term hospitalization. ${ }^{2}$ Latzer et al., found that the median length of stay prior to the time of S. maltophilia isolation was 40 days (range 23.5-59 days) in critically ill children. Their patients who did not survive were those who, at the time of culture acquisition, had already had a prior longer length of stay (50 days in non-survivors vs. 18.5 in those who survived, $\mathrm{p}=0.002) .{ }^{10}$ As a result, $S$. maltophilia should be considered a breakthrough infection including bacteremia in the pediatric population receiving broad-spectrum antibiotics during a prolonged hospital stay. ${ }^{4}$

The presence of an indwelling CVC is a risk factor for biofilm formation with direct or indirect contact on various surfaces, such as glass, plastics, and host tissues. Biofilm formation is a significant feature of S. maltophilia. ${ }^{1,2}$ The significance of catheter removal in the treatment of catheterrelated S. maltophilia BSI is unclear as well as some studies have suggested removal of the central line

TABLE 2. Patient bacteremia duration, treatment, antibiogram, and prognosis

\begin{tabular}{|c|c|c|c|c|c|c|}
\hline Patient & $\begin{array}{l}\text { Duration of } \\
\text { bacteremia* }\end{array}$ & Treatment & $\begin{array}{l}\text { Trimethoprim- } \\
\text { sulfamethoxazole }\end{array}$ & Levofloxacin & $\underset{* \star}{\text { Ciprofloxacin }}$ & Outcome \\
\hline 1 & 4 days & $\begin{array}{l}\text { Trimethoprim- } \\
\text { sulfamethoxazole }\end{array}$ & s & S & S & Discharged \\
\hline 2 & 8 days & Ciprofloxacin & s & $\mathrm{s}$ & S & Discharged \\
\hline 3 & $\begin{array}{l}\text { No control blood } \\
\text { culture }\end{array}$ & Ciprofloxacin & - & s & S & $\begin{array}{c}\text { Exitus } \\
\left({ }^{* * *} 5 \text { th day) }\right.\end{array}$ \\
\hline 4 & 12 days & $\begin{array}{l}\text { Trimethoprim- } \\
\text { sulfamethoxazole }\end{array}$ & s & S & s & $\begin{array}{c}\text { Exitus } \\
\left({ }^{*+*} 20 \text { th day }\right)\end{array}$ \\
\hline 5 & $\begin{array}{c}\text { No control blood } \\
\text { culture }\end{array}$ & Ciprofloxacin & $\mathrm{R}$ & $\mathrm{R}$ & - & $\begin{array}{c}\text { Exitus } \\
(* * 2 \text { 2nd day })\end{array}$ \\
\hline 6 & 9 days & Ciprofloxacin & s & S & s & Discharged \\
\hline 7 & 23 days & $\begin{array}{l}\text { Trimethoprim- } \\
\text { sulfamethoxazole } \\
\text { and ciprofloxacin }\end{array}$ & s & S & S & Discharged \\
\hline 8 & 6 days & $\begin{array}{l}\text { Trimethoprim- } \\
\text { sulfamethoxazole } \\
\text { and ciprofloxacin }\end{array}$ & $\mathrm{s}$ & I & - & Discharged \\
\hline 9 & 5 days & Ciprofloxacin & s & I & s & Discharged \\
\hline 10 & 25 days & Levofloxacin & s & $\mathrm{s}$ & $\mathrm{R}$ & Discharged \\
\hline 11 & $\begin{array}{l}\text { No control blood } \\
\text { culture }\end{array}$ & No & s & 1 & $\mathrm{R}$ & $\begin{array}{c}\text { Exitus } \\
\left({ }^{* *} 1 \text { st day }\right)\end{array}$ \\
\hline 12 & 7 days & Ciprofloxacin & s & S & s & Discharged \\
\hline & \multicolumn{6}{|c|}{$\begin{array}{l}\text { "Duration between first positive and negative cultures } \\
\text { **Antimicrobial susceptibility, S: Sensitive R: Resistant, I: Intermediate } \\
{ }^{* *} \text { After first positive culture }\end{array}$} \\
\hline
\end{tabular}


in addition to antibiotic therapy..$^{2,11}$

S. maltophilia may be associated with polymicrobial infections. Araoka et al., reported a higher mortality rate related to bacteremia in cases of mixed infection with enterococci. ${ }^{2,12}$ Furthermore, polymicrobial infections with S. maltophilia and other organisms, such as Enterobacter cloacae, may result in the emergence of antibiotic resistance, as this species carries resistance plasmids or transposons. ${ }^{13}$

S. maltophilia commonly causes respiratory tract infections and bacteremia. Approximately $11 \%$ of cystic fibrosis patients are colonized by S. maltophilia. ${ }^{2}$ S. maltophilia is also a cause of ventilator-associated pneumonia. ${ }^{1}$ The prevalence of S. maltophilia infections was $1.2 \%$ among children $\leq 7$ years and $1.4 \%$ among children $\leq 18$ years old in SENTRY studies conducted in 1998-2003 and in 2004. ${ }^{1}$ Pneumonia with bacteremia is associated with a higher mortality when compared with catheter-related bloodstream infection. S. maltophilia may cause pulmonary hemorrhage, especially in adult patients with hematologic diseases. ${ }^{14}$ In the present study, S. maltophilia was regarded to have a direct role in the deaths of three of the fatal cases. The deaths of two of the nonsurvivors were associated with pneumonia. S. maltophilia can adhere to human bronchial epithelial cells, and biofilms can be formed on lung cells. ${ }^{2}$ S. maltophilia also produces StmPr1 protease, which has been implicated in the damage and destruction of lung tissue resulting from S. maltophilia invasion. ${ }^{15}$

Studies on the treatment options for S. maltophilia infections, including bacteremia, are limited in the pediatric population..$^{10}$ In the current study, the most frequently used antimicrobial agent was ciprofloxacin, followed by trimethoprim/sulfamethoxazole (TMP / SMX). Latzer et al., found that a combination of ciprofloxacin and TMP / SMX significantly extended the survival time and that ciprofloxacin provided a significantly longer survival time than TMP/SMX when either was given as a monotherapy in their critically ill pediatric patients. $^{10}$

The present study is one of the few studies of S. maltophilia bacteremia in childhood. The clinical features of $S$. maltophilia bacteremia in our study were similar to those reported in the current literature. Notably, two of our nonsurvivors were associated with pneumonia. This opportunistic pathogen should therefore be viewed as a breakthrough agent for bacteremia in pediatric patients with underlying disease who have been exposed to broad-spectrum antibiotics during long-term hospital stay.

\section{REFERENCES}

1. Chang YT, Lin CY, Chen YH, Hsueh PR. Update on infections caused by Stenotrophomonas maltophilia with particular attention to resistance mechanisms and therapeutic options. Front Microbiol. 2015; 6:893.

2. Brooke JS. Stenotrophomonas maltophilia: an emerging global opportunistic pathogen. Clin Microbiol Rev. 2012; 25(1):2-41.

3. Jeon YD, Jeong WY, Kim MH, Jung IY, et al. Risk factors for mortality in patients with Stenotrophomonas maltophilia bacteremia. Medicine (Baltimore). 2016; 95(31):e4375.

4. Ebara H, Hagiya H, Haruki Y, Kondo E, Otsuka F. Clinical Characteristics of Stenotrophomonas maltophilia Bacteremia: A Regional Report and a Review of a Japanese Case Series. Intern Med. 2017; 56(2):137-42.

5. Wu PS, LuCY, Chang LY,Hsueh PR, etal.Stenotrophomonas maltophilia bacteremia in pediatric patients-- a 10-year analysis. J Microbiol Immunol Infect. 2006; 39(2):144-9.

6. Furuichi M, Ito K, Miyairi I. Characteristics of Stenotrophomonas maltophilia bacteremia in children. Pediatr Int. 2016; 58(2):113-8.

7. Clinical and Laboratory Standards Insitute. Performance Standards for Antimicrobial Susceptebility Testing: Nineteenth Informational Supplement. M100-S19. Wayne, Pa: CLSI; 2009.

8. Clinical and Laboratory Standards Insitute. Performance Standards for AntimicrobialSusceptebility Testing: TwentyFirst Informational Supplement. M100-S21. Wayne, Pa: CLSI; 2011.

9. CDC. Bloodstream Infection Event (Central LineAssociated Bloodstream Infection and Non-central-LineAssociated-Bloodstream-Infection). 2019. [Acceso: 24 de octubre de 2019]. Disponible en: https: / / www.cdc.gov/ nhsn/pdfs / pscmanual/4psc_clabscurrent.pdf

10. Tokatly Latzer I, Paret G, Rubinstein M, Keller N, et al. Management of Stenotrophomonas maltophilia Infections in Critically Ill Children. Pediatr Infect Dis J. 2018;37(10):9816.

11. Naidu P, SmithS. A review of 11 years of Stenotrophomonas maltophilia blood isolates at a tertiary care institute in Canada. Can J Infect Dis Med Microbiol. 2012; 23(4):165-9.

12. Araoka H, Baba M, Yoneyama A. Risk factors for mortality among patients with Stenotrophomonas maltophilia bacteremia in Tokyo, Japan, 1996-2009. Eur J Clin Microbiol Infect Dis. 2010; 29(5):605-8.

13. Juhász E, Krizsán G, Lengyel G, Grósz G, et al. Infection and colonization by Stenotrophomonas maltophilia: antimicrobial susceptibility and clinical background of strains isolated at a tertiary care centre in Hungary. Ann Clin Microbiol Antimicrob. 2014; 13:333.

14. Kim SH, Cha MK, Kang CI, Ko JH, et al. Pathogenic significance of hemorrhagic pneumonia in hematologic malignancy patients with Stenotrophomonas maltophilia bacteremia: clinical and microbiological analysis. Eur J Clin Microbiol Infect Dis. 2019; 38(2):285-95.

15. Windhorst S, Frank E, Georgieva DN, Genov N, et al. The major extracellular protease of the nosocomial pathogen Stenotrophomonas maltophilia: characterization of the protein and molecular cloning of the gene. J Biol Chem. 2002; 277(13):11042-9. 


\section{ANNEX}

\section{DEFINITIONS}

Previous antimicrobial therapy: intramuscular and intravenous administrations of antibacterial drugs more than $48 \mathrm{~h}$ within 30 days before detecting blood culture-positivity. ${ }^{1}$

Duration of bacteremia: the time in days between the first positive and the first negative blood culture. $^{1}$

Polymicrobial bacteremia: identification of two or more bacterial species in multiple blood culture bottles of samples collected within $24 \mathrm{~h} .{ }^{2}$

Concomitant infection: isolation of an organism other than S. maltophilia within 1 week of the first positive blood culture. ${ }^{2}$

If $S$. maltophilia or an identical causative pathogen was isolated from a patients' clinical specimen (including contaminated blood culture) within 30 days before the onset of bacteremia, it was recorded as a previous isolation of $S$. maltophilia or previous isolation of an identical pathogen. ${ }^{3}$

Persistently positive blood culture: isolation of $S$. maltophilia from subsequent blood cultures taken 2 days from the treatment. ${ }^{4}$

Immunosuppressed patient: who receiving chemotherapy or radiotherapy for malignancies, immunosuppressive therapies with a daily dose $\geq 20 \mathrm{mg}$ prednisolone-equivalent steroid ( $\geq 14$ days) monoclonal antibodies, antimetabolite drugs or T cell inhibitors within the preceding 30 days of the positive blood culture, neutropenia. ${ }^{4}$

CVC and mechanical ventilation: use within $48 \mathrm{~h}$ before first positive blood culture. ${ }^{4}$

Community-acquired bacteremia: bacteremia that occurred within 48 hours of admission and in patients who did not meet the criteria for healthcare-associated bacteremia. 2,5

Healthcare-associated bacteremia: bacteremia that occurs in a patient who has stayed in a nursing home, has been admitted to a hospital within the previous month, received hemodialysis, or has been treated as an outpatient with intravenous antibiotics or chemotherapy within the previous 2 weeks. ${ }^{2,5}$

Hospital-acquired bacteremia: a positive blood culture obtained from patients hospitalized for $>48$ hours after admission. 2,5

Sepsis and septic shock: according to the international consensus definitions. ${ }^{6}$

Removal of the CVC: when this was performed no later than 5 days after blood cultures were drawn. $^{2}$
Empirical therapy: administration of antibiotics at the onset of symptoms of bacteremia. ${ }^{7}$

Adequate treatment: targeted administration of at least one antimicrobial agent to which S. maltophilia was susceptible in vitro ${ }^{2}$ and appropriate empiric therapy was defined as microorganism susceptibility to one of several antimicrobial agents administered within 72 hours after the onset of bacteremia. ${ }^{8}$

Response to treatment: either as the resolution of all clinical manifestations of bacteremia or by a follow-up blood culture that was negative for S. maltophilia during treatment. ${ }^{9}$

Failure of treatment: persistence or progression of the clinical signs and symptoms of bacterial infection and / or blood cultures that were positive for S. maltophilia during treatment. ${ }^{9}$

S. maltophilia related death: it occurred within 7 days of the positive culture and if clinical signs and symptoms of the infections were documented in the medical record when the patient died and was considered that the patient would not have died in the absence of bacteraemia. ${ }^{10}$

\section{REFERENCES}

1. Kagen J, Zaoutis TE, McGowan KL, Luan X, Shah SS. Bloodstream infection caused by Stenotrophomonas maltophilia in children. Pediatr Infect Dis J. 2007; 26(6):508-12.

2. Jeon YD, Jeong WY, Kim MH, Jung IY, et al. Risk factors for mortality in patients with Stenotrophomonas maltophilia bacteremia. Medicine (Baltimore). 2016; 95(31):e4375.

3. Kim SH, ChoSY, Kang CI, Seok H, et al. Clinical predictors of Stenotrophomonas maltophilia bacteremia in adult patients with hematologic malignancy. Ann Hematol. 2018;97(2):343-50.

4. Furuichi M, Ito K, Miyairi I. Characteristics of Stenotrophomonas maltophilia bacteremia in children. Pediatr Int. 2016; 58(2):113-8.

5. CDC. Bloodstream Infection Event (Central LineAssociated Bloodstream Infection and Non-central Line Associated Bloodstream Infection). 2019. [Acceso: 25 de octubre de 2019]. Disponible en: https: / / www.cdc.gov/ nhsn/pdfs / pscmanual/4psc_clabscurrent.pdf

6. Mathias B, Mira JC, Larson SD. Pediatric sepsis. Curr Opin Pediatr. 2016; 28(3):380-7.

7. Lakatos B, Jakopp B, Widmer A, Frei R, et al. Evaluation of treatment outcomes for Stenotrophomonas maltophilia bacteraemia. Infection. 2014; 42(3):553-8.

8. Wang $\mathrm{CH}$, Lin JC, Lin HA, Chang FY, et al. Comparisons between patients with trimethoprim-sulfamethoxazolesusceptible and trimethoprim-sulfamethoxazole-resistant Stenotrophomonas maltophilia monomicrobial bacteremia: A 10-year retrospective study. J Microbiol Immunol Infect. 2016; 49(3):378-86.

9. Boktour M, Hanna H, Ansari S, Bahna B, et al. Central venous catheter and Stenotrophomonas maltophilia bacteremia in cancer patients. Cancer. 2006; 106(9):1967-73.

10. Mutlu M, Yilmaz G, Aslan Y, Bayramoglu G. Risk factors and clinical characteristics of Stenotrophomonas maltophilia infections in neonates. J Microbiol Immunol Infect. 2011; 44(6):467-72 